\title{
Numerical analysis for muscle forces supporting the cervical spine
}

\author{
S. Tadano \& T. Katagiri \\ Division of Human Mechanical Systems and Design, \\ School of Engineering, Hokkaido University, Sapporo, Japan
}

\begin{abstract}
This paper describes a method of numerical analysis to simulate the muscle forces supporting the cervical spine in motion from flexion to extension. Each of twenty-one muscles was modelled by single or multiple lines from the origin area to the insertion point on the skeleton. These locations were measured by the $\mathrm{X}$-ray CT and MRI images of a male volunteer. The posture of cervical vertebrae in motion was also measured by the plane X-ray examination. Under static conditions, twenty-four equilibrium equations on force and moment were formulated to solve 222 unknown parameters of muscle and joint reaction forces. The optimization analysis using the SQP method was applied to calculate this indeterminate problem. The objective function was expressed as the sum of the squares of muscle force divided by the physiological cross sectional area of each muscle. Results obtained by the analysis have been matched to the measured electromyographic activity.

Keywords: biomechanics, muscle force, numerical analysis, cervical spine, optimization, cooperative motion.
\end{abstract}

\section{Introduction}

The biomechanical function of the cervical spine is to protect the spinal cord, support the skull, and enable diverse head movement. Knowledge of biomechanics provides an essential framework for understanding the consequences of injury and disorders [1]. A complex system of ligaments, tendons and muscles helps to support and stabilize the cervical spine (fig. 1). Muscles contract and relax in response to nerve impulses originating in the brain. Every degree of freedom of motion in the cervical spine is actuated and 
controlled by more than two muscles. Some muscles work in pairs or as antagonists. This means when a muscle contracts, the opposing muscle relaxes. It is impossible to measure experimentally the force of every muscles acting in motion of the head and neck. Therefore, many biomechanical models have proposed to simulate the muscle activities in the neck motion. Helleur et al [2] reported a sagittal plane mathematical model to simulate the muscular response of the cervical spine in some specific tasks. Dulhunty [3] presented a simplified graphical model for evaluating force transmission in the upright static cervical spine. Snijders et al [4] used a kinematic model with eight links. Authors have proposed an optimization method to solve the force of many muscles in cooperative motion with the synergist and the antagonist on forearm [5], index finger [6] and shoulder [7]. In this paper, applying our method to the head and neck motion, the computational method is described to analyze muscle forces supporting the cervical spine. Each of twenty-one muscles was modelled by a single or multiple straight lines that stretch between the point of origin and the point of insertion on the skeleton. The locations of these points were measured from the X-ray CT and the MRI image of a male volunteer. In order to analyse the relationship between every muscle activities and occlusal surface angle from a neutral position, the posture of each cervical vertebra during flexion to extension was measured by the plane X-ray examination. Cervical vertebra is subjected by muscle forces, reaction forces in facet joints and an intervertebral disc, vertebral moment, and the weight of head and neck. Under static conditions, twenty-four equilibrium equations on force and moment were formulated to solve 222 unknowns of muscle and joint reaction forces. The optimization analysis of the SQP method was applied to calculate this indeterminate problem. The objective function was selected as the sum of squares of muscle force divided by PCSA (physiological cross sectional area) of each muscle. Results of the superficial neck muscles obtained by the analysis were checked to the measured electromyographic activity.
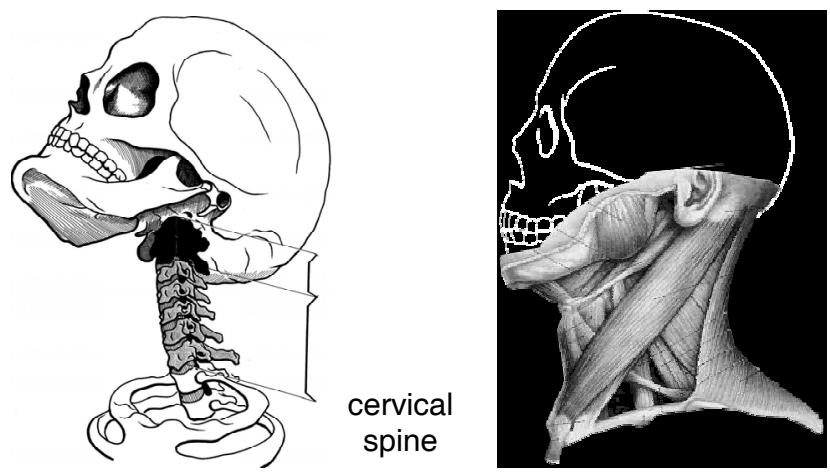

Figure 1: Human neck anatomy, skeletals and muscles in cervical spine. 


\section{Biomechanical modelling and motion analysis}

The authors et al [5-7] have proposed an optimization technique for the static equilibrium analysis to simulate the muscular response of the musculo-skeletal system. It is assumed that the skeleton consists of rigid bodies articulated by joints and held together by muscles, where ligaments and tendons are not considered, as they does not actuate the cervical segments. To apply this method to the cervical spine, a precise anatomical geometry of the skeleton must be constructed as digital data from skull to $\mathrm{C} 1-\mathrm{C} 7$ vertebra. Both X-ray CT image and MRI image of a volunteer (24 y.o. male) were used to measure the attachment points of muscle on the skeleton. The location of all attachment points of muscles was determined from the CT image as shown in fig. 2. The muscles are considered to be a collection of muscle strands that are represented by vectors. Each vector runs from a point of origin to a point of insertion. Table 1 lists muscles selected in this study and their location of origin and insertion. The abbreviation shows an anatomical name of muscle; RCA (rectus capitis anterior), RCL (rectus capitis lateralis), LgCp (longus capitis), SA (scalenus anterior), SM (scalenus medius), $\mathrm{LgCl}$ (longus colli), SCM (sternocleidomastoideus), RCMa (rectus capitis posterior major), RCMi (rectus capitis posterior minor), OCS (obliquus capitis superior), OCI (obliquus capitis inferior), $\mathrm{LmCp}$ (longissimus capitis), $\mathrm{SpCp}$ (splenius capitis), $\mathrm{SmCp}$ (semispinalis capitis), $\mathrm{LmCv}$ (longissimus cervicis), $\mathrm{SpCv}$ (splenius crevicis), $\mathrm{SmCv}$ (semispinalis cervicis), SP (scalenus posterior), IC (iliocostalis crevicis), $\operatorname{Tr}$ (trapezius), LS (levator scapulae). To analyse the behavior of muscle activities in motion of flexion to extension, a sagittal posture data of each vertebra in the cervical spine was measured by using a plane X-ray photograph in flexion-extension ( -40 to $75 \mathrm{deg}$ ) as shown in fig. 3. From these motion data and the anatomical geometry, the local coordinate positions of the origin and insertion points of every muscle around the cervical spine were determined at every five deg of the occlusal surface angle, and transformed to $3 \mathrm{D}$ data in the global coordinates.

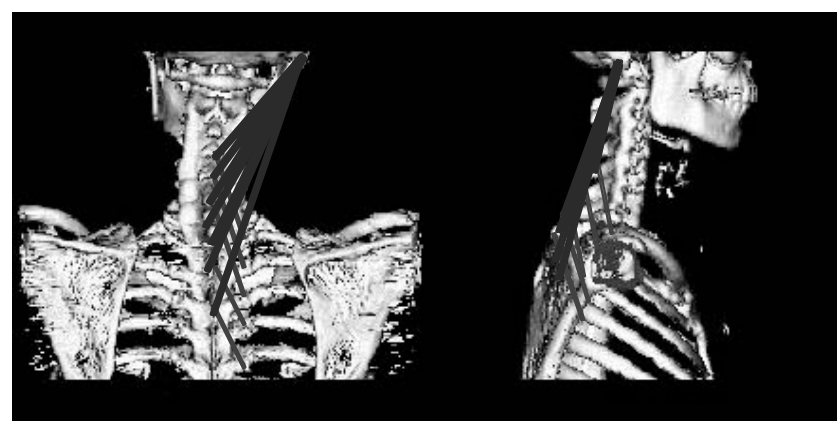

Figure 2: $\quad \mathrm{X}$-ray CT image of cervical spine. 


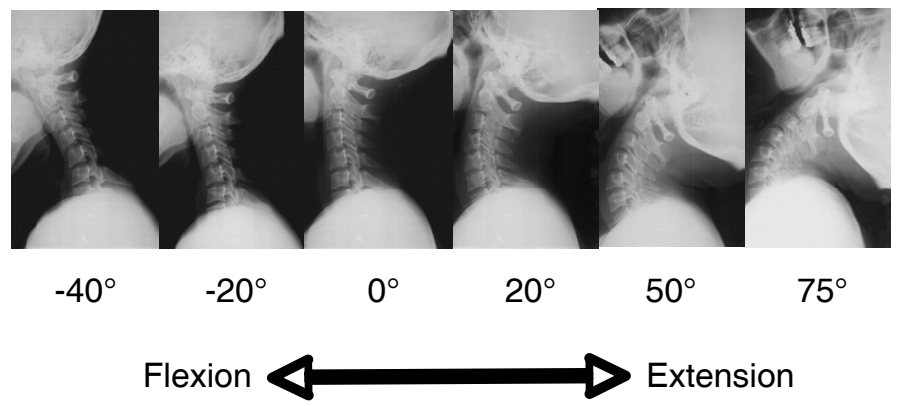

Figure 3: $\quad \mathrm{X}$-ray photos in motion from flexion to extension.

Table 1: $\quad$ Muscle used in the model and its origin and insertion point.

\begin{tabular}{|c|c|c|c|c|}
\hline & Muscle & Origin & Insertion & Group \\
\hline & IC & Rib 4, 5 & C4-C6 t.p.-p.t. & Ext-E \\
\hline & LmCp & C5-T3 t.p. & m.p. & $\mathrm{H}-\mathrm{E}$ \\
\hline & $\mathrm{LmCv}$ & T3,4 t.p. & C2-C5 t.p.-p.t. & $\mathrm{N}-\mathrm{E}$ \\
\hline $\mathrm{SpCp}$ & Medial & C4-T3 s.p. & Near m.p. & $\mathrm{H}-\mathrm{E}$ \\
\hline & Lateral & T1-T4 & m.p. & \\
\hline $\mathrm{SpCv}$ & & T4 & C1-C2 t.p. & $\mathrm{H}-\mathrm{E}$ \\
\hline & SmCp & $\begin{array}{l}\text { C3-C7 a.p. } \\
\text { T1-T6 t.p. }\end{array}$ & i.n.l.-s.n.l. & $\mathrm{H}-\mathrm{E}$ \\
\hline & SmCv & T1-T5 t.p. & C3-C7 s.p. & $\mathrm{N}-\mathrm{E}$ \\
\hline & $\mathrm{RCMn}$ & C1 p.t. & i.n.l. & $\mathrm{H}-\mathrm{E}$ \\
\hline & $\mathrm{RCMi}$ & C2 s.p. & i.n.I. & $\mathrm{H}-\mathrm{E}$ \\
\hline & $\mathrm{RCA}$ & C1 t.p.-a.t. & Bas-occ & $\mathrm{H}-\mathrm{F}$ \\
\hline & OCS & C1 t.p. & i.n.I.-up & $H-E$ \\
\hline & $\mathrm{OCl}$ & C2 s.p. & C1 t.p. & $\mathrm{H}-\mathrm{E}$ \\
\hline & $\mathrm{RCL}$ & C1 I.m. & Bas-occ & $\mathrm{H}-\mathrm{F}$ \\
\hline & LgCp & C3-C6 t.p.-a.t. & Bas-occ & $\mathrm{H}-\mathrm{F}$ \\
\hline $\mathrm{LgCl}$ & vertical & C3-C5 t.p.-a.t. & C1a.t. & $\mathrm{N}-\mathrm{F}$ \\
\hline & superior oblique & T1-T3 ant v.b. & C6t.p.-a.t. & \\
\hline & inferior oblique & C5 ant v.b. & C1-C4 ant v.b. & \\
\hline & & C6 & C1-C4 ant v.b. & \\
\hline & & $\mathrm{C} 7$ & C1-C4 ant v.b. & \\
\hline & & T1 & C1-C4 ant v.b. & \\
\hline & & T2 & C1-C4 ant v.b. & \\
\hline & SA & C3-C6 t.p.-a.t. & Rib1 & $\mathrm{N}-\mathrm{F}$ \\
\hline & SM & C1-C7 t.p.-p.t. & Rib1-mem & $\mathrm{N}-\mathrm{F}$ \\
\hline & SP & C5-C7 t.p.-p.t. & Rib2 & Ext-F \\
\hline SCM & SMO & str & m.p.-s.n.l. & $\mathrm{N}-\mathrm{F}$ \\
\hline & CM & clv & m.p. & \\
\hline & $\mathrm{CO}$ & clv & s.n.l. & \\
\hline & $\operatorname{Tr}$ & occ pr, s.n.l. & clv & Ext-E \\
\hline & LS & $\begin{array}{l}\text { C1-C6 s.p. } \\
\text { C1-C4 t.p.-p.t. }\end{array}$ & Med & Ext-E \\
\hline
\end{tabular}




\section{Numerical method}

When the head and neck move very slowly by themselves, an external load to be applied to the cervical spine is their own weights. Under static condition, the moments must balance at each cervical joint to satisfy the equilibrium law. This is a simplified assumption to get a solution of the analysis. As shown in fig. 4, each cervical vertebra is subjected by muscle force $F$, reaction force $R$ in facet joints and intervertebral disc, and vertebral moment $M$ around the gravity center, and the weight $W$ of head and the upper portions of the neck. A muscle was modeled as a single or multiple straight lines from the insertion to the origin. Numbers of line element for a muscle was determined as according to the area of attachment, and listed in table 2. LgCp (inferior oblique) has the maximum twenty elements. A total is 102 muscle elements for twenty-one muscles. The equilibrium equations on force and moment were formulated by using the threedimensional rigid body model of each vertebra as eqns (1) and (2), respectively,

$$
\begin{gathered}
\sum F_{i}+\sum R_{i}-\sum R_{i-1}+W_{i}=0 \\
\sum\left(r f_{i} \times F_{i}\right)+\sum\left(r r_{i} \times R_{i}\right)-\sum\left(r r_{i-1} \times R_{i-1}\right)+r w_{i} \times W_{i}+M_{i}-M_{i-1}=0
\end{gathered}
$$

where subscript-i in above equations means a cervical joint level, and $r f, r r$ and $r w$ are a position vector of the muscle attachment, the joint center and the gravity center, respectively. The loading condition is the weights of head $(40 \mathrm{~N})$ and $\mathrm{C1}$ C6 vertebra (each1.5N). Twenty-four equations were obtained as threedimensional components from eqns (1) and (2). Whereas, there is a total of 222 unknown parameters on muscle and joint reaction forces to be solve. This is a typical static indeterminate problem. Because not every muscle force can be calculated directly from these equations, an optimization analysis of the SQP (Sequential Quadratic Programming) method was used to solve these equations. The objective function was selected as the total summation of squares of muscle force $F \mathrm{i}$ divided by physiological cross sectional area of muscle (PCSAi) [8,9], as eqn (3).

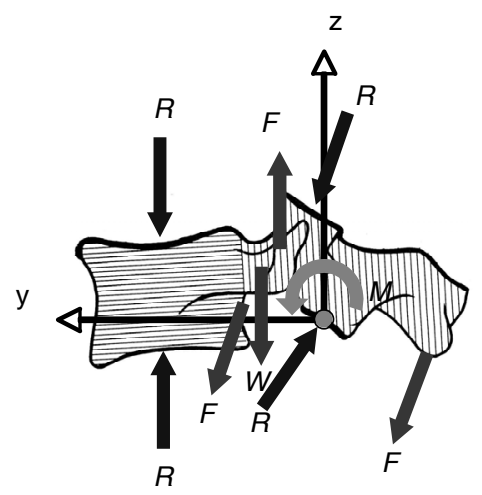

Figure 4: Rigid body model of a vertebra and exposed in loading distribution. 


$$
f=\sum\left(F_{i} / P C S A_{i}\right)^{2} \rightarrow \text { minimize }
$$

The PSCA value was defined as eqn (4) using muscle mass $m$, pennation angle $\theta$, muscle density $\left(1.06 \mathrm{~g} / \mathrm{cm}^{3}\right) \rho$ and normalized fascicle length $l$.

$$
P C S A=\frac{m \times \cos \theta}{\rho \times l}
$$

The values obtained at each muscle are also listed in table 2. Eqn (3) was minimized in calculation process under the constraint condition that all muscle forces are tensile and all reaction forces of joints and discs are always compressive. At the same time, the muscle intensities doses not exceed the peak force as shown in table 2 . The peak force was calculated from the multiplication of a limit stress $\left(35 \mathrm{~N} / \mathrm{cm}^{2}\right)$ of muscle and PSCA.

\begin{tabular}{|c|c|c|c|c|c|}
\hline \multicolumn{2}{|c|}{ Muscles } & $\mathrm{N}$ & $\begin{array}{l}\text { Peak force } \\
(\mathrm{N})\end{array}$ & $\begin{array}{l}\text { PCSA } \\
\left(\mathrm{cm}^{2}\right)\end{array}$ & $\begin{array}{c}\mathrm{PCSA} / \mathrm{N} \\
\left(\mathrm{cm}^{2}\right)\end{array}$ \\
\hline \multicolumn{2}{|l|}{ IC } & 3 & 20.00 & 0.57 & 0.190 \\
\hline \multicolumn{2}{|l|}{ LmCp } & 6 & 31.00 & 0.89 & 0.148 \\
\hline \multicolumn{2}{|l|}{$\mathrm{LmCv}$} & 4 & 20.00 & 0.57 & 0.143 \\
\hline $\mathrm{SpCp}$ & $\begin{array}{l}\text { medial } \\
\text { lateral }\end{array}$ & $\begin{array}{l}4 \\
3\end{array}$ & 99.40 & 2.84 & 0.406 \\
\hline $\mathrm{SpCv}$ & & 11 & 49.70 & 1.42 & 0.129 \\
\hline SmCp & & 2 & 126.00 & 3.60 & 1.800 \\
\hline $\mathrm{SmCv}$ & & 5 & 63.00 & 1.80 & 0.360 \\
\hline $\mathrm{RCMa}$ & & 1 & 17.50 & 0.50 & 0.500 \\
\hline RCMi & & 1 & 32.55 & 0.93 & 0.930 \\
\hline $\mathrm{RCL}$ & & 1 & 8.75 & 0.25 & 0.250 \\
\hline OCS & & 1 & 36.05 & 1.03 & 1.030 \\
\hline $\mathrm{OCl}$ & & 1 & 45.15 & 1.29 & 1.290 \\
\hline $\mathrm{RCA}$ & & 1 & 8.75 & 0.25 & 0.250 \\
\hline \multirow[t]{2}{*}{ LgCp } & & 4 & 32.20 & 0.92 & 0.230 \\
\hline & vertical & 3 & 10.00 & 0.29 & 0.095 \\
\hline \multirow[t]{2}{*}{ LgCl } & sup. obli. & 3 & 10.00 & 0.29 & 0.095 \\
\hline & inf. obli. & 20 & 10.00 & 0.29 & 0.014 \\
\hline SA & & 4 & 50.75 & 1.45 & 0.363 \\
\hline SM & & 7 & 70.00 & 2.00 & 0.286 \\
\hline \multirow[t]{2}{*}{ SP } & & 3 & 54.25 & 1.55 & 0.517 \\
\hline & SMO & 1 & 65.10 & 1.86 & 1.860 \\
\hline \multirow[t]{2}{*}{ SCM } & $\mathrm{CM}$ & 1 & 32.55 & 0.93 & 0.930 \\
\hline & $\mathrm{CO}$ & 1 & 32.55 & 0.93 & 0.930 \\
\hline $\operatorname{Tr}$ & & 7 & 68.60 & 1.96 & 0.280 \\
\hline LS & & 4 & 76.30 & 2.18 & 0.545 \\
\hline
\end{tabular}

Table 2: Numbers of line element, peak force and PCSA of each muscle.

\section{Electromyographic investigation}

The experimental investigation was done to measure the firing patterns of cervical spine muscles of a volunteer performing in motion of flexion to 
extension. The muscular patterns were measured from EMG activity collected by surface electrodes. It is impossible to measure all muscles around the cervical spine. At specific muscles of SCM, SPL, TPZ (up) and TPZ (mid) running in the surface layer under the skin, the patterns was measured from the EMG. Surface EMG electrodes (bioload 45352V, NEC) were attached on the portion of these muscles of a volunteer, who is the same male of 24 y.o. as the CT and MRI image investigation. In flexion of $-40,-20 \mathrm{deg}$ and extension of 25, 50, $75 \mathrm{deg}$, the posture at every angle was hold in 5 seconds and then integrated EMG was measured by Polygraph 360SYSTEM (NEC). Five measurements were done at the same condition. Data were cut below $10 \mathrm{~Hz}$ and were integrated at every 100 msec. The mean value of integrated EMG was used to the comparison with the simulation results.

\section{Results and discussion}

\subsection{Motion at each cervical joint}

Fig. 5 shows the variation of cervical vertebra angle at each vertebra in the motion of flexion to extension (-40 to $75 \mathrm{deg})$, from a neutral position $(0 \mathrm{deg})$. The abscissa axis is the occlusal surface angle defined as an index of whole movement of the head and neck. A characteristic movement of each vertebra in motion was observed from this figure. Each vertebra did not move in a uniform manner. In flexion, C5-6 and C4-5 joints in the mid-lower cervis moved more, but the top joint of SKL-C1 and the bottom joint of C6-C7 moved in an opposite direction of extension. In extension, SKL-C1 moved with the largest angle, but $\mathrm{C} 1-\mathrm{C} 2$ and $\mathrm{C} 2-\mathrm{C} 3$ of the upper joint did not move.

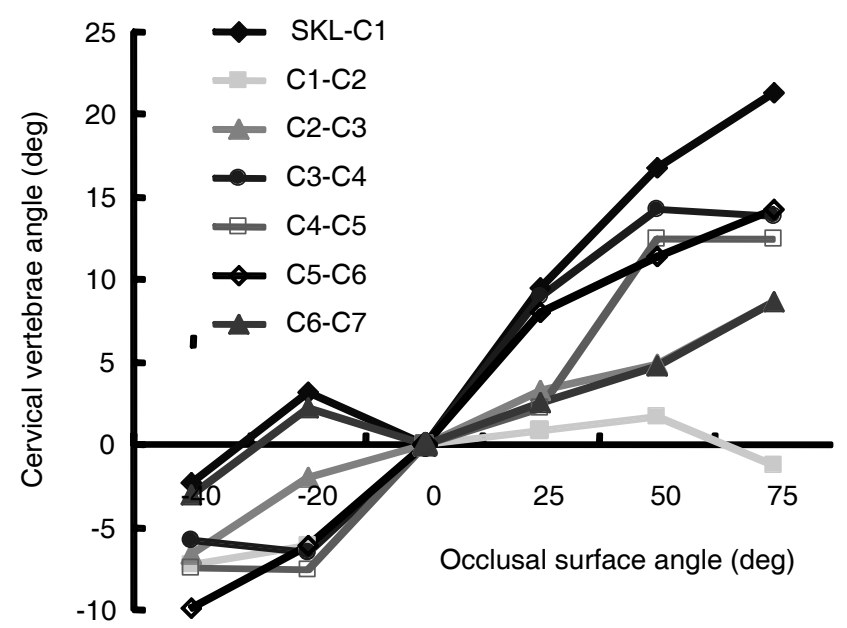

Figure 5: Movement of each vertebra in flexion to extension. 


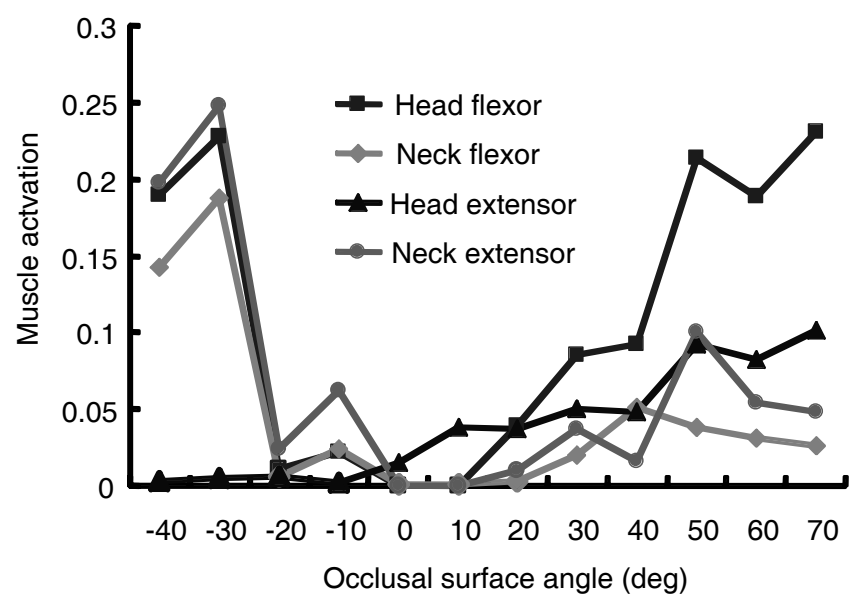

Figure 6: Muscle activation of four muscle groups; head flexor, neck flexor, head extensor and neck extensor.

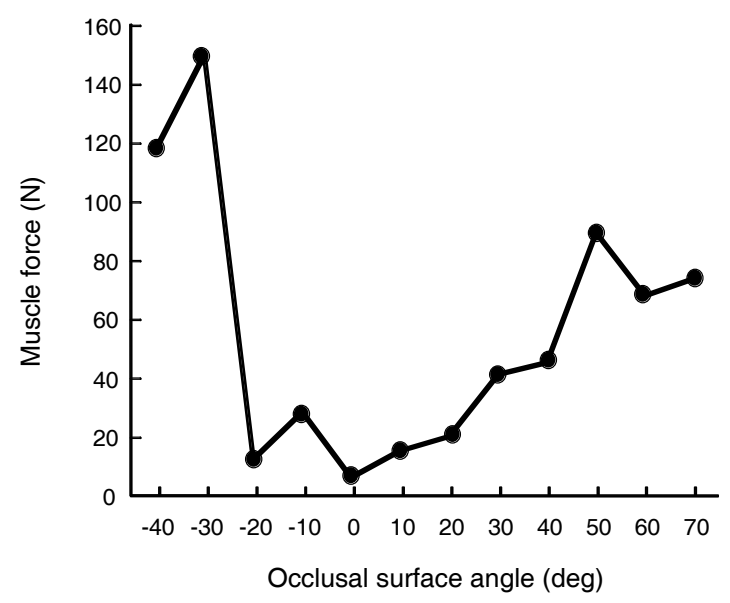

Figure 7: Total forces of cervical muscles during neck motion.

\subsection{Simulation results}

The variation of twenty-one muscle forces in motion could be obtained from the simulation by this method. To avoid complexity of the results, muscles around cervical spine were classified into four functional groups, which also lists in table 1. The group of head flexor (H-F in table 1) is RCA, RCL and $\mathrm{LgCp}$, the neck flexor (N-F) is $\mathrm{LgCl}, \mathrm{SA}, \mathrm{SM}$ and SCM, the head extensor (H-E) is LmCp, $\mathrm{SpCp}, \mathrm{SpCv}, \mathrm{SmCp}, \mathrm{RCMn}, \mathrm{RCMi}, \mathrm{OCS}$ and OCI, and the neck extensor (N-E) is $\mathrm{LmCv}$ and $\mathrm{SmCv}$. Fig. 6 shows the muscle activation of each group, where the values was expressed as the summation of muscle force in the same group. In 
flexion stage of the head and neck, not only the head flexor and the neck flexor but also the neck extensor muscle groups generated larger muscle force, whereas the head extensor acted as antagonists. In extension stage, every muscle groups generated muscle force. Muscle force of the head flexor showed the largest value at $-30 \mathrm{deg}$. Total summation of muscle force is shown in fig. 7. The maximum activation occurred in flexion stage of $-30 \mathrm{deg}$. When the head and neck is zero angle of a neutral position, the muscle activation showed also in almost zero. The value increased gradually with increasing the occlusal surface angle in extension.
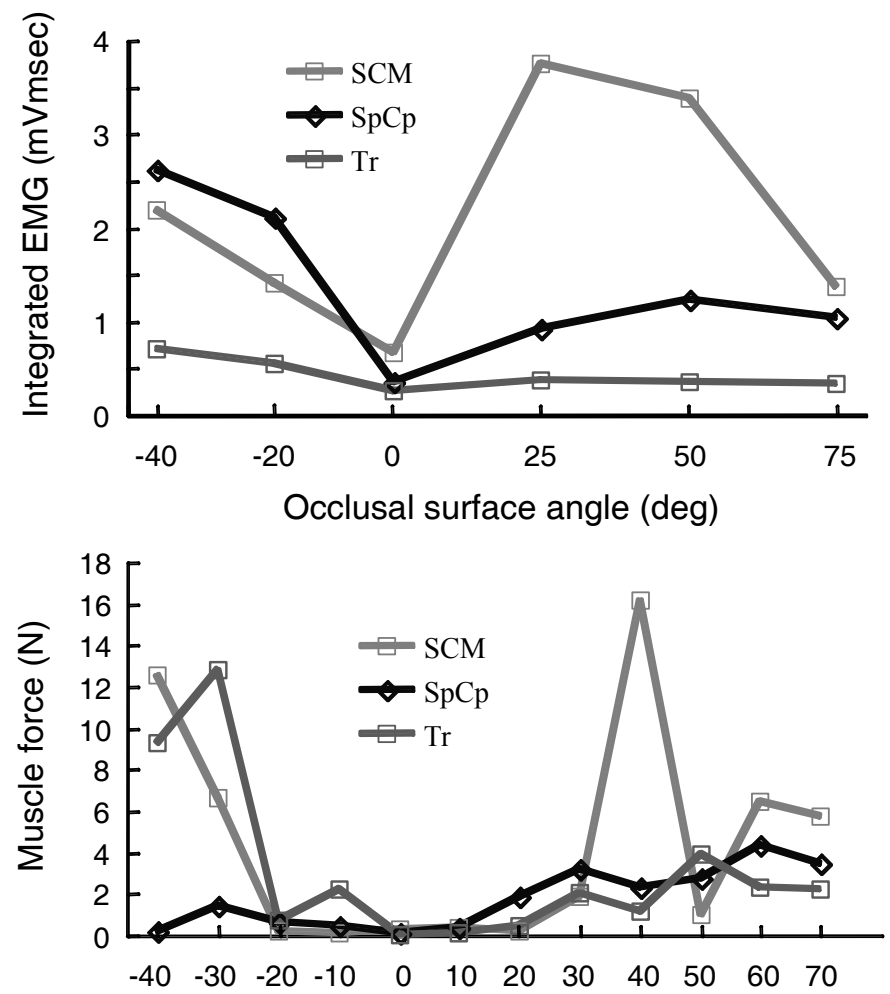

Occlusal surface angle (deg)

Figure 8: Comparison of simulation results with electromyogaphic results on $\mathrm{SCM}, \mathrm{SpCp}$ and $\mathrm{Tr}$ muscles.

\subsection{Comparison with EMG}

Because only three muscles of SCM, SpCp and Tr could be measured by the EMG experiment, the simulation results were also represented in accordance with these muscles. In fig. 8, the upper figure is the result of integrated EMG and the lower is the simulation result. It is clear from the figure that EMG measurements cannot be use for an accurate quantitative evaluation of muscle 
activities. Therefore, the development of computer simulation is much important as this work. In comparison with both figures, a broad qualitative agreement could be confirmed. In flexion stage, SCM and SpCp activate but only SCM activates in extension stage. Therefore, the validity of this method was checked from the comparison of both figures, even if the very complex system of activation in motion of the head and neck.

\section{Concluding remarks}

From this work, some notable results could be obtained on the muscle force acting in motion of the head and neck. This will give one of effective biomechanical informations for muscle physiology and clinical orthopaedics.

\section{References}

[1] Krag, M. H., Biomechanics of the cervical spine (Chapter 45). The Adult Spine: Principles and Practice, Editor-in-Chief J. W. Frymoyer, Raven Press: New York, pp. 929-945, 1991.

[2] Helleur, C., Gracovetsky, S., Farfan, H. \& Wingrowicz, E., Modelling of the muscular response of the cervical spine. Mathematical Modelling, 6, pp. 443-462, 1985.

[3] Dulhunty, J. DC., A simplified conceptual model of the human cervical spine for evaluating force transmission in upright static posture. Journal of Manipulative and Physiological Therapeutics, 25(5), pp. 306-317, 2002.

[4] Snijders, C. J., Hoek van Dijke, A. \& Roosch, E. R., A biomechanical model for the analysis of the cervical spine in static postures. Journal of Biomechanics, 24(9), pp. 783-792, 1991.

[5] Tadano, S., Yoshinari, S., Kimura, H. \& Ukai, T., A numerical analysis of muscle force in a human upper limb during flexion of the elbow joint. Transactions of JSME, Series A, 62(604), pp. 202-208, 1996.

[6] Tadano, S. \& Fukada, K., Numerical analysis of musulotendinous forces and joint reaction forces during motion of index finger. Transactions of JSME, Series A, 67(653), pp. 168-174, 2001.

[7] Oizumi, N., Tadano, S., Narita, Y., Suenaga, N., Iwasaki, N. \& Minami, A., Numerical analysis of the muscle force during abduction of the shoulder using the three-dimensional skeletal muscle model. Transactions of JSME, Series A, 69(677), pp. 77-83, 2003.

[8] Yamaguchi, G., Sawa, U., Moran, D. W., Fessler, M. J. \& Winters, J. M., A Survey of human musculotendon actuator parameters, Multiple Muscle Systems, Springer-Verlag, pp. 717-773, 1990.

[9] Vasavada, A. N., Li, S. \& Delp, S. L., Influence of muscle morphometry and moment arms on the moment-generating capacity of human neck muscles, Spine, 23(4), pp. 412-422, 1998. 